\title{
A METHOD OF PROVING CERTAIN THEOREMS RELATING TO RATIONAL FUNCTIONS WHICH ARE ADJOINT TO AN ALGEBRAIC EQUATION FOR A GIVEN VALUE OF THE INDEPENDENT VARIABLE*
}

BY

J. C. FIELDS

In the sixth chapter of the writer's book $\dagger$ on the theory of the algebraic functions it was proved that a rational function of $(z, v)$, which is adjoint for the value $z=\infty$ relatively to an algebraic equation $F(z, v)=0$, must be of degree $\overline{<} N-1$ and that the degree of the element involving $v^{n-1}$ must be $\overline{<} n-1$, where $N$ is the degree of the equation supposed to be written in the form

$$
F(z, v)=v^{n}+F_{n-1} v^{n-1}+\cdots+F_{0}=0 .
$$

At the place cited the equation was integral. This however is not necessary and is not here assumed. The first purpose of the present paper is to prove the theorem in question by a shorter and simpler method. To this end it will be necessary to recall some of the terms made use of in the earlier chapters of the book referred to and it will also be convenient for the sake of completeness to reproduce a few of the results there given.

The equation (1) we may write in the form

$$
F(z, v)=\left(v-z^{a_{1}} P_{1}\right) \cdots\left(v-z^{a_{n}} P_{n}\right)=0,
$$

where $P_{1}, \cdots, P_{n}$ are power-series in $1 / z$ whose exponents may be integral or fractional. They are however none of them negative and in each of the power-series the constant term is different from 0 . The branches group themselves into a number $r$ of cycles and the orders of coincidence of the branches of these cycles, each with the product of the $n-1$ conjugate factors $v-z^{a_{5}} P_{s}$, we indicate by the symbols $\mu_{1}, \cdots, \mu_{r}$ respectively. Any rational function of $(z, v)$ we say is adjoint relatively to the equation, for the

* Presented to the Society, December, 1910.

+ Theory of the algebraic functions of a complex variable, MAYER and MÜLLER (Bierlin, 1906). 
value $z=\infty$, if its orders of coincidence with the branches of the several cycles do not fall short of the numbers $\mu_{1}-1+1 / \nu_{1}, \cdots, \mu_{r}-1+1 / \nu_{r}$ respectively, where $\nu_{1}, \cdots, \nu_{r}$ are the orders of the several cycles.

If in the product (2) the exponents $\alpha_{k+1}, \cdots, \alpha_{n}$ are those which are greater than 1 , we evidently have

$$
N=k+\alpha_{k+1}+\cdots+\alpha_{n} .
$$

It is then clear that the degree of the product of any $n-1$ of the factors $v-z^{a_{1}} P_{1}, \cdots, v-z^{a_{n}} P_{n}$ is $\overline{<} N-1$. The same will also be true of any product $\left(v-z^{\beta_{1}} Q_{1}\right) \cdots\left(v-z^{\beta_{n-1}} Q_{n-1}\right)$ in which $Q_{1}, \cdots, Q_{n-1}$ are powerseries in $1 / z$ not involving positive powers of $z$, and in which the exponents $\beta_{1}, \cdots, \beta_{n-1}$ have for values some $n-1$ of the $n$ numbers $\alpha_{1}, \cdots, \alpha_{n}$.

Let us now consider a rational function of the form

$$
v^{n-1}+R_{n-2} v^{n-2}+\cdots+R_{0},
$$

in which the coefficients $R_{n-2}, \cdots, R_{0}$ are rational functions of $z$. Writing this as a product

$$
\left(v-z^{\beta_{1}} Q_{1}\right) \cdots\left(v-z^{\beta_{n-1}} Q_{n-1}\right)
$$

we see that, by choice of the power-series $Q$ and the exponents $\beta$, we cannot simultaneously increase indefinitely the orders of coincidence of the product with more than $n-1$ of the $n$ branches $v-z^{a} \cdot P_{s}=0$ of the equation $F(z, v)=0$. Furthermore the product can have with $n-1$ of these branches such indefinitely high orders of coincidence, only when the $n-1$ factors $v-z^{\beta} Q_{s}$ correspond severally to the $n-1$ branches in question and coincide each to an indefinitely high order with one of these branches. Now any rational function of $(z, v)$, of degree $n-1$ in $v$, can evidently be written in the form

$$
R(z, v)=z^{-k} Q\left(v-z^{\beta_{1}} Q_{1}\right) \cdots\left(v-z^{\beta_{n-1}} Q_{n-1}\right),
$$

where $Q$ is a power-series in $1 / z$ beginning with a constant term which is different from 0 and where $k$ is an integer which may happen to be positive, negative or zero. From what precedes we see that such rational function cannot be given indefinitely high orders of coincidence with all the $n$ branches $v-z^{\mathrm{c}_{1}} P_{1}=0, \cdots, v-z^{\mathrm{an}_{n}} P_{n}=0$ simultaneously, unless we take $k$ indefinitely large.

Assuming now that the orders of coincidence of the function (6) with all $n$ branches increase indefinitely, and therewith that $k$ also increases indefinitely, we can readily show that the degree of the function (6) must decrease indefinitely. Let us consider the order of coincidence of the function (6) with the branch $v-z^{a} \cdot P_{s}=0$. This order of coincidence is evidently equal to the sum of the orders of coincidence of the product 
and the product

$$
\left(v-z^{\beta_{1}} Q_{1}\right) \cdots\left(v-z^{\beta_{p}}\right)
$$

$$
z^{-k} Q\left(v-z^{\beta_{p+1}} Q_{p+1}\right) \cdots\left(v-z^{\beta_{n-1}} Q_{n-1}\right)
$$

with the branch in question. Here we shall assume our notation to be so chosen that the exponents $\beta_{p+1}, \cdots, \beta_{n-1}$ are those among the exponents $\beta$ which are $>\alpha_{8}$. If the order of coincidence of the branch $v-z^{a_{s}} P_{8}=0$ with the product (6) becomes indefinitely great its order of coincidence with one of the two products (7) and (8) must become indefinitely great. Its order of coincidence with the latter product however is evidently

$$
k-\beta_{p+1}-\cdots-\beta_{n-1} .
$$

If this number becomes indefinitely great the degree in $(z, v)$ of the product (8) must plainly become indefinitely small. It will then follow also that the degree of the product (6) must decrease indefinitely, since the degree of the product (7) is evidently limited. Indefinite increase of the order of coincidence of the branch $v-z^{a_{s}} P_{s}=0$ with the product (6) then brings with it indefinite decrease of the degree of this product or indefinite increase in the order of coincidence of the branch with the product (7). The latter alternative, however, requires indefinite increase in the order of coincidence of the branch with one of the factors of the product (7) and therefore with one of the factors of the product (5). Indefinitely large increase in the orders of coincidence of all $n$ branches $v-z^{a_{1}} P_{1}=0, \cdots, v-z^{a_{n}} P_{n}=0$ with the function (6) will then require either indefinite decrease in the degree of the function or indefinite increase in the orders of coincidence of the $n$ branches, each with a corresponding one of the $n-1$ factors in the product (5). The latter alternative is, however, obviously impossible. It follows therefore that the degree of the function (6) must decrease indefinitely if its orders of coincidence with the $n$ branches increase indefinitely.

In the above the rational function $R(z, v)$ has been assumed to contain a term involving the power $v^{n-1}$. If however the degree in $v$ of the function is $<n-1$ we readily see, on replacing $n-1$ by $n-t-1$ in (6), that our preceding argument applies to the product so obtained and that its degree must diminish indefinitely if its orders of coincidence with the $n$ branches increase indefinitely. The same result can otherwise be deduced on considering the product $v^{t} R(z, v)$ in the case where $R(z, v)$ is of degree $n-t-1$ in $v$. Indefinite increase in the orders of coincidence of the function $R(z, v)$ with the $n$ branches brings with it indefinite increase in the orders of coincidence of the product $v^{t} R(z, v)$ with these branches, and therefore indefinite decrease in the degree of this product-since it involves the power $v^{n-1}$-and consequently also indefinite decrease in the degree of the factor $R(z, v)$. In any case, 
then, indefinite increase in the orders of coincidence of a rational function of $(z, v)$ with all $n$ branches of the fundamental equation corresponding to the value $z=\infty$ brings with it indefinite decrease in the degree of the function - supposed to be expressed in its reduced form.

Assuming for the moment that the lettering of the $n$ branches of the equation has been so ordered that the $\nu_{s}$ branches $v-z^{a_{1}} P_{1}=0, \cdots, v-z^{a_{v_{0}}} P_{v_{s}}=0$ constitute the cycle of order $\nu_{s}$, let us consider the product

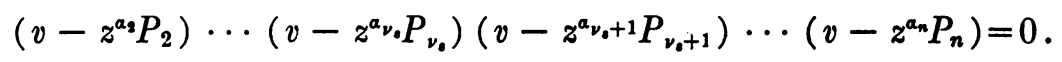

Here we have of course $\alpha_{1}=\alpha_{2}=\cdots=\alpha_{\nu_{\mathrm{s}}}$. The orders of coincidence of the product with all the branches excepting $v-z^{a_{1}} P_{1}=0$ are infinite and with this branch its order of coincidence is $\mu_{s}$.

From the product (10) we derive another product

$$
\left(v-z^{a_{2}} Q_{2}\right) \cdots\left(v-z^{a_{v}} Q_{v_{s}}\right)\left(v-z^{a_{v_{s}+1}} Q_{v_{t}+1}\right) \cdots\left(v-z^{a_{n}} Q_{n}\right),
$$

where the $Q$ 's are obtained on discarding terms of higher order in the series $P_{2}, \cdots, P_{n}$. We may assume terms of like order to be discarded in the case of all series belonging to the same cycle. The product

$$
\bar{R}(z, v)=\left(v-z^{a_{v_{0}}+1} Q_{v_{0}+1}\right) \cdots\left(v-z^{a_{n}} Q_{n}\right)
$$

will then be a rational function of $(z, v)$ and its orders of coincidence with the $n-\nu_{s}$ branches $v-z^{a_{v_{s}+1}} P_{\nu_{t}+1}=0, \cdots, v-z^{a_{n}} P_{n}=0$ we may assume to be as large as we like. In the $Q$ 's of the $\nu_{s}-1$ factors of the product

$$
\left(v-z^{a_{2}} Q_{2}\right) \cdots\left(v-z^{\left.a_{v_{s}} Q_{v_{s}}\right)}\right.
$$

we suppose terms of sufficiently high order to be retained, in order that the order of coincidence of the product (11) with the branch $v-z^{\alpha_{1}} P_{1}=0$ may be equal to $\mu_{s}$, while its orders of coincidence with the $\nu_{s}-1$ branches $v-z^{a_{2}} P_{2}=0, \cdots, v-z^{\alpha_{v}} P_{\nu_{s}}=0$ are all greater than $\mu_{8}$.

The product (11) can evidently be written in the form

$$
B(z, v)=\left\{B_{0}(z, v)+B_{1}(z, v) z^{-1 / v_{0}}+\cdots B_{v_{0}-1}(z, v) z^{-1+1 / v_{0}}\right\} \cdot \bar{R}(z, v) .
$$

Representing the $\nu_{s}$ th roots of unity by $e_{1}=1, e_{2}, \cdots, e_{\nu_{\mathrm{e}}}$, construct the $\nu_{\mathrm{s}}$ functions

$$
\begin{aligned}
& B^{(t)}(z, v)=\left\{B_{0}(z, v)+\right. B_{1}(z, v) e_{t} z^{-1 / v_{s}} \\
&\left.+\cdots+B_{v_{s}-1}(z, v) e_{t}^{v_{t}-1} z^{-1+1 / v_{s}}\right\} \bar{R}(z, v), \\
& t=1,2, \cdots, \nu_{s} .
\end{aligned}
$$

We derive the function $B^{(t)}(z, v)$ from the function $B(z, v)$ on replacing in this function $z^{-1 / v_{t}}$ by $e_{t} z^{-1 / v_{s}}$. The branch $v-z^{a_{t}} P_{t}=0$ may be assumed 
to be derived in like manner from the branch $v-z^{a_{1}} P_{1}=0$. The order of coincidence of the function $B^{(t)}(z, v)$ with the corresponding branch $v-z^{a_{t}} P_{t}=0$ is then evidently $\mu_{s}$, and its orders of coincidence with the other branches of the same cycle are greater than $\mu_{s}$. At the same time we may assume the orders of coincidence of the function with the branches of the remaining cycles to be as large as we please, because of the presence of the factor $\vec{R}(z, v)$ in its expression as given in (14).

From (14) it evidently follows that the function

$$
\sum_{t=1}^{\nu_{s}} e^{\nu_{c}-\sigma} B^{(t)}(z, v)=\nu_{s} B_{\sigma}(z, v) z^{-\sigma / v_{*}} \cdot \bar{R}(z, v)
$$

has $\mu_{s}$ as its order of coincidence with each of the branches of the cycle of order $\nu_{s}$, while its orders of coincidence with the branches of the remaining cycles are indefinitely large. Here $\sigma$ may have any one of the values $0,1, \cdots$, $v_{s}-1$. The orders of coincidence of the rational functions

$$
B_{0}(z, v) \cdot \bar{R}(z, v), \quad B_{1}(z, v) \cdot \bar{R}(z, v), \cdots B_{v_{0}-1}(z, v) \cdot \bar{R}(z, v)
$$

with the branches of the cycle of order $\nu_{s}$ will then have the values

$$
\mu_{s}, \mu_{s}-\frac{1}{\nu_{s}}, \cdots, \mu_{s}-\frac{\nu_{s}-1}{\nu_{s}}
$$

respectively. At the same time the orders of coincidence of these functions with the branches of the remaining $r-1$ cycles may be supposed to be as large as we please. What we are here giving is merely an explicit repetition for the value $z=\infty$ of what has already been given for the value $z=a$ in the earlier chapters of the writer's book already referred to. The repetition however is necessary for the purpose of pointing out the limitation on the degrees of the functions which here present themselves in connection with certain sets of orders of coincidence. We note that the degree in $(z, v)$ of the product (11) is $\overline{<} N-1$, and the same will therefore be the case for the functions $B^{(t)}(z, v)$ in (14), and consequently for the functions $B_{\sigma}(z, v) \cdot z^{-\sigma / v_{v}} \bar{R}(z, v)$ in (15), and therefore also for the $\nu_{s}$ rational functions in (16).

Now let us construct a system of $r$ sets of functions like those in (16), each set corresponding to a different one of the $r$ cycles. These $n=\sum_{s=1}^{r} \nu_{s}$ functions we shall indicate by the notation

$$
B_{s, \sigma}(z, v) \cdot R_{s}(z, v), \quad s=1,2, \cdots, r ; \quad \sigma=0,1, \cdots, \nu_{s}-1 .
$$

The function $B_{s, \sigma}(z, v) \cdot R_{s}(z, v)$ has $\mu_{s}-\sigma / \nu_{s}$ as its order of coincidence with the branches of the cycle of order $\nu_{s}$, while its orders of coincidence with the branches of the other cycles are indefinitely great. The degree of the function is also $\bar{\gtrless} N-1$. 
If now $\mu_{1}^{\prime}, \cdots, \mu_{r}^{\prime}$ represent any set of adjoint orders of coincidence for the branches of the cycles here in question, we can write them in the form

$$
m_{1}+\mu_{1}-\sigma_{1} / \nu_{1}, \cdots, m_{r}+\mu_{r}-\sigma_{r} / \nu_{r},
$$

where the numbers $m_{s}$ are positive integers or 0 and where a number $\sigma_{s}$ has one of the $\nu_{s}$ values $0,1, \cdots, \nu_{s}-1$. A rational function which possesses precisely this set of orders of coincidence is evidently furnished by the sum

$$
\sum_{s=1}^{r} \lambda_{s} z^{-m_{s}} B_{s, \sigma_{s}}(z, v) \cdot R_{s}(z, v),
$$

where the coefficients $\lambda_{s}$ are constants all of which are different from 0 . We see also, from what precedes, that the degree of this function is $\bar{\gtrless} N-1$.

We are now in a position to prove that a rational function of $(z, v)$ which is adjoint for the value $z=\infty$ must be of degree $\bar{\gtrless} N-1$. For assume that there exists a certain rational function $R^{\prime}(z, v)$, of degree $>N-1$, which possesses a specific set of adjoint orders of coincidence $\mu_{1}^{\prime}, \cdots, \mu_{r}^{\prime}$, for the branches of the $r$ cycles corresponding to the value $z=\infty$. By proper choice of the coefficients $\lambda_{s}$ in the sum

$$
R^{\prime \prime}(z, v)=R^{\prime}(z, v)+\sum_{s=1}^{r} \lambda_{s} z^{-m_{s}} B_{s, \sigma_{s}}(z, v) \cdot R_{s}(z, v)
$$

we can then evidently give the function $R^{\prime \prime}(z, v)$ a set of adjoint orders of coincidence $\mu_{1}^{\prime \prime}, \cdots, \mu_{r}^{\prime \prime}$, each of which is greater than the corresponding number in the set $\mu_{1}^{\prime}, \cdots, \mu_{r}^{\prime}$. Here it is plain that each one of the $r$ coefficients $\lambda_{s}$ is determined independently of the other $r-1$ coefficients. For, apart from $R^{\prime}(z, v)$, the only other element on the right-hand side of (21) which has $m_{s}+\mu_{s}-\sigma_{s} / \nu_{s}$ as its order of coincidence with the branches of the cycle of order $\nu_{s}$ is the element with $\lambda_{s}$ as coefficient. Since, by hypothesis, the function $R^{\prime}(z, v)$ is of degree $>N-1$, and since the remainder of the expression on the right-hand side of (21) is known to be of degree $\overline{<} N-1$, it follows that the function $R^{\prime \prime}(z, v)$ must be of the same degree as $R^{\prime}(z, v)$. Assuming, then, that there exists a rational function $R^{\prime}(z, v)$ of degree $>N-1$, which possesses a set of adjoint orders of coincidence $\mu_{1}^{\prime}, \cdots, \mu_{r}^{\prime}$ for the branches of the cycles corresponding to the value $z=\infty$, it follows that there exists also a rational function $R^{\prime \prime}(z, v)$, of the same degree as $R^{\prime}(z, v)$, which possesses a set of adjoint orders of coincidence $\mu_{1}^{\prime \prime}, \cdots, \mu_{r}^{\prime \prime}$, each one of which is greater than the corresponding member of the former set.

By successive application of the process described in what precedes, 'we could evidently construct a sequence of functions $R^{\prime}(z, v), R^{\prime \prime}(z, v)$, $R^{\prime \prime \prime}(z, v), \cdots$ all of the same degree, and each one possessing a set of adjoint 
orders of coincidence for the value $z=\infty$, whose members severally exceed the corresponding orders of coincidence of the next preceding function in the sequence. We could then construct a rational function of the same degree as $R^{\prime}(z, v)$ which would possess indefinitely great orders of coincidence with the branches of all $r$ cycles corresponding to the value $z=\infty$. This result however is at variance with the fact established in the earlier part of this paper, that a rational function can have simultaneously indefinitely high orders of coincidence with all $n$ branches corresponding to the value $z=\infty$, only when the degree of the rational function is decreased indefinitely. The assumption, then, of the existence of a rational function of degree $>N-1$, which is adjoint for the value $z=\infty$, leads to contradiction. A rational function of $(z, v)$, adjoint for the value $z=\infty$, must therefore be of degree $\bar{\gtrless} N-1$.

Looking back over the preceding pages, we see that the power $v^{n-1}$ does not appear in a term of degree $>n-1$ in the product (11), nor therefore in the functions $B^{(t)}(z, v)$ in (14), nor yet in the rational functions (16). The power $v^{n-1}$, then, will not present itself in a term of degree $>n-1$ in any of the functions in (18) - and in fact the only ones among these functions in which such power of $v$ will present itself at all are the $r$ functions in which the suffix $\sigma$ has the value 0 . We see furthermore that in (20) a term involving $v^{n-1}$ can never be of degree $>n-1$, and that therefore if the function $R^{\prime}(z, v)$ which appears in (21) involves $v^{n-1}$ in a term of degree $>n-1$, the same will be true of the function $R^{\prime \prime}(z, v)$ and of a sequence of functions $R^{\prime \prime \prime}(z, v)$, $\ldots$, constructed in the manner already considered. From this would follow the existence of a rational function of degree $>n-1$, whose orders of coincidence with the $n$ branches corresponding to the value $z=\infty$ are all indefinitely great. This however is impossible, and the assumption of the existence of a rational function $R^{\prime}(z, v)$, adjoint for the value $z=\infty$ and involving $v^{n-1}$ in a term of degree $>n-1$, leads to contradiction. If then the power $\boldsymbol{v}^{n-1}$ presents itself in a rational function which is adjoint for the value $z=\infty$,

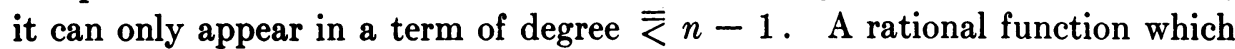
is adjoint for the value $z=\infty$ must therefore be of degree $\bar{\gtrless} N-1$, and can only involve the power $v^{n-1}$ in a term of degree $\bar{\gtrless} n-1$.

We shall now assume that our fundamental algebraic equation (1) is integral. The $n$ branches corresponding to a finite value $z=a$ we shall suppose to group themselves into a number $r$ of cycles of orders $\nu_{1}, \nu_{2}, \cdots, \nu_{r}$, respectively, and by $\mu_{1}, \mu_{2}, \cdots, \mu_{r}$ we shall indicate the orders of coincidence of the branches of these cycles, each with the product of the remaining $n-1$ branches. We can show that an integral rational function of $(z, v)$ must be divisible by the factor $z-a$, if its orders of coincidence with the branches of the $r$ cycles corresponding to the value $z=a$ are simultaneously greater 
than the numbers $\mu_{1}, \cdots, \mu_{r}$. This theorem has been proved in the writer's book* already cited, by the method of the "deformation of a product." We shall prove it by the method employed in obtaining the results in what precedes.

In chapter $\mathrm{V}$ of the book just quoted, integral rational functions were constructed, possessing as their orders of coincidence with the branches of the $r$ cycles here in question an arbitrarily assigned set of adjoint orders of coincidence. Where these orders of coincidence were simultaneously greater than the numbers $\mu_{1}, \cdots, \mu_{r}$ respectively, the actually constructed functions were in all cases divisible by the factor $z-a$. Let $\mu_{1}^{\prime}, \mu_{2}^{\prime}, \cdots, \mu_{r}^{\prime}$ represent such a set of adjoint orders of coincidence, and let $(z-a) g_{1}(z, v)$ be one of the actually constructed functions just referred to, which possesses precisely these orders of coincidence with the branches of the cycles corresponding to the value $z=a$. Here $g_{1}(z, v)$ is an integral rational function which may or may not itself be divisible by the factor $z-a$.

Now suppose, if possible, that there exists an integral rational function $G_{1}(z, v)$ which is not divisible by $z-a$, and which possesses $\mu_{1}^{\prime}, \mu_{2}^{\prime}, \cdots, \mu_{r}^{\prime}$ as its orders of coincidence with the branches of the several cycles. By proper choice of the constant $c_{1}$ in the sum

$$
G_{2}(z, v)=G_{1}(z, v)+c_{1}(z-a) g_{1}(z, v)
$$

we can evidently make the order of coincidence of the function $G_{2}(z, v)$ with the branches of an arbitrarily chosen one of the $r$ cycles exceed the corresponding number $\mu_{s}^{\prime}$. Among the orders of coincidence $\mu_{1}^{\prime \prime}, \mu_{2}^{\prime \prime}, \cdots, \mu_{r}^{\prime \prime}$ of the function $G_{2}(z, v)$ with the branches of the several cycles, no order is less than the corresponding number in the set $\mu_{1}^{\prime}, \mu_{2}^{\prime}, \cdots, \mu_{r}^{\prime}$, and some one order is greater than the corresponding number in this set-the order here in question corresponding to an arbitrarily selected one of the $r$ cycles. Furthermore the integral rational function $G_{2}(z, v)$ is plainly not divisible by $z-a$.

By repeated applications of the above process, it would evidently be possible to construct an integral rational function $G(z, v)$, which is not divisible by $z-a$, and whose orders of coincidence with the branches of the $r$ cycles are all as great as we will - always of course on the assumption of the existence of the function $G_{1}(z, v)$ as described above. We can, however, in contradiction with this, readily prove, that with indefinite increase in the orders of coincidence of an integral rational function with all $n$ branches corresponding to the value $z=a$ the function will become divisible by an indefinitely high power of $z-a$. To prove this theorem we evidently only have to replace $1 / z$ by the element $z-a$, in the argument by which in the earlier part of the paper we have proved indefinite decrease in the degree of a rational function

* See chapters III and IV. 
to follow as a consequence of indefinite increase in the orders of coincidence of the function with all $n$ branches corresponding to the value $z=\infty$.

The assumption, then, of the existence of an integral rational function $G_{1}(z, v)$, which is not divisible by $z-a$ and whose orders of coincidence $\mu_{1}^{\prime}, \mu_{2}^{\prime}, \cdots, \mu_{r}^{\prime}$ are severally greater than the corresponding numbers in the set $\mu_{1}, \mu_{2}, \cdots, \mu_{r}$, leads to contradiction of the theorem whose proof we have just indicated. It follows therefore that any integral rational function of $(z, v)$, whose orders of coincidence with the branches of the $r$ cycles corresponding to the value $z=a$ are simultaneously greater than the numbers $\mu_{1}, \mu_{2}, \cdots, \mu_{r}$ respectively, must be divisible by $z-a$.

As a corollary of the above theorem follows, that a rational function which is adjoint for the value $z=a$ must, in its reduced form, be integral with regard to the element $z-a$. If, namely, a rational function $R(z, v)$ has orders of coincidence with the branches of the several cycles, which do not fall short of the numbers

$$
\mu_{1}-1+\frac{1}{\nu_{1}}, \quad \mu_{2}-1+\frac{1}{\nu_{2}}, \cdots, \quad \mu_{r}-1+\frac{1}{\nu_{r}},
$$

it follows that the orders of coincidence of the product $(z-a) R(z, v)$, with the branches of the several cycles are simultaneously greater than the numbers $\mu_{1}, \mu_{2}, \cdots, \mu_{r}$ respectively. Writing $R(z, v)$ in the form of a quotient $G(z, v) / g(z)$ where $G(z, v)$ and $g(z)$ are integral and have no factor in common, it is clear that the denominator $g(z)$ cannot have $z-a$ as a factor. For in that case the product $g(z) \cdot R(z, v)=G(z, v)$ would evidently have orders of coincidence with the branches of the $r$ cycles which are simultaneously greater than the numbers $\mu_{1}, \mu_{2}, \cdots, \mu_{r}$ respectively, and from this would follow that $G(z, v)$ must also have $z-a$ as a factor, contrary to our hypothesis that $G(z, v)$ and $g(z)$ have no factor in common.

Still assuming the fundamental algebraic equation to be integral, we can utilize our method to prove the following theorem: If an integral rational function has adjoint orders of coincidence with $n-1$ of the $n$ branches corresponding to the value $z=a$, its order of coincidence with the remaining branch must also be adjoint.

The theorem is self-evident if the branch in question belongs to a cycle of order greater than 1 , for the orders of coincidence of a rational function with the branches of a cycle are all the same. Suppose however that $v-P_{1}=0$ is a simple branch and suppose, if possible, that there exists an integral rational function $G_{1}(z, v)$ whose order of coincidence with this branch falls short of adjointness, while its orders of coincidence with the remaining $n-1$ branches are all adjoint. Construct an integral rational function $g_{1}(z, v)$ possessing 
a set of adjoint orders of coincidence $\mu_{1}^{\prime}, \mu_{2}^{\prime}, \cdots, \mu_{r}^{\prime}$ which, apart from the order of coincidence with the branch $v-P_{1}=0$, are precisely the same as the orders of coincidence of the function $G_{1}(z, v)$ with the branches in question. By proper choice of the constant $c_{1}$ in the sum

$$
G_{2}(z, v)=G_{1}(z, v)+c_{1} g_{1}(z, v)
$$

we can then evidently obtain an integral rational function $G_{2}(z, v)$, whose order of coincidence with the branch $v-P_{1}=0$ is the same as that of the function $G_{1}(z, v)$ with this branch, while its orders of coincidence with the branches of the remaining cycles nowhere fall short of the orders of coincidence of the function $G_{1}(z, v)$ with the branches of these cycles, while, in the case of one of these $r-1$ cycles at least, and that one arbitrarily chosen, the order of coincidence of the function $G_{2}(z, v)$ exceeds that of the function $G_{1}(z, v)$.

In the manner just indicated we could construct a succession of integral rational functions $G_{1}(z, v), G_{2}(z, v), G_{3}(z, v), \ldots$ all of which possess the same order of coincidence with the branch $v-P_{1}=0$, while each successive one exceeds all those which precede it in its order of coincidence with the branches of some one of the remaining $r-1$ cycles, arbitrarily chosen, and does not for any cycle fall short of the orders of coincidence of the preceding functions. We could evidently thus construct an integral rational function $G(z, v)$ whose order of coincidence with the branch $v-P_{1}=0$ would be the same as that of the original function $G_{1}(z, v)$ with this branch, while its orders of coincidence with the remaining $n-1$ branches would be as large as we might please.

Since the order of coincidence of $G(z, v)$ with the branch $v-P_{1}=0$ falls short of adjointness, it cannot have as factor an indefinitely high power of $z-a$. On dividing $G(z, v)$ through by any power of $z-a$ which it may happen to have as factor we then obtain an integral rational function which is not divisible by $z-a$. The orders of coincidence of this function with $n-1$ of the branches are still indefinitely great, while its order of coincidence with the remaining branch $v-P_{1}=0$ falls short of adjointness. The same set of orders of coincidence* will then also be those of an integral rational function in which the coefficient of $v^{n-1}$ is not divisible by $z-a$, and therefore at the same time the orders of coincidence of a product of the form

$$
\left(v-Q_{1}\right)\left(v-Q_{2}\right) \cdots\left(v-Q_{n-1}\right),
$$

in which the $Q$ 's are power-series in $z-a$. Since the orders of coincidence of this product with the $n-1$ branches $v-P_{2}=0, \cdots, v-P_{n}=0$ are all

* See first two pages of chapter III in book already cited. 
indefinitely great, each one of these branches must have an indefinitely high order of coincidence with some one factor of the product, and the order of coincidence of the branch $v-P_{1}=0$ with this product will therefore be the same as its order of coincidence with the product

$$
\left(v-P_{2}\right)\left(v-P_{3}\right) \cdots\left(v-P_{n}\right) .
$$

The order of coincidence of the branch $v-P_{1}=0$ with the product (24) then is adjoint, contrary to our deduction that the order of coincidence in question for this product fell short of what was requisite to adjointness. The contradiction is a consequence of our assumption of the existence of an integral rational function $G_{1}(z, v)$, whose order of coincidence with the branch $v-P_{1}=0$ falls short of adjointness, while its orders of coincidence with the remaining $n-1$ branches are all adjoint. It follows that such a function does not exist, and consequently that any integral rational function which is adjoint for $n-1$ of the branches corresponding to a finite value $z=a$ must also be adjoint for the remaining branch.

UNIVERSITY OF TORONTO. 